\title{
Synchronized Attractors and phase entrained with cavity loss of the coupled laser's map
}

\author{
M. Abdul, ${ }^{1,2, *}$ Altaf Ur Rahman, ${ }^{3, *}$ Chen Minjing, ${ }^{4, *}$ Jehan Akbar, ${ }^{5,6, *}$ F. Saif, ${ }^{7}$ and Zhiyou Zhang ${ }^{8}, *$ \\ ${ }^{1}$ Department of Physical Science and Technology, Sichuan University, Chengdu, Sichuan, China \\ ${ }^{2}$ Department of Modern Physics, University of Science and Technology of China, Hefei, China \\ Division of Quantum Physics and Quantum Information \\ ${ }^{3}$ Department of Physics Riphah International University Lahore, Pakistan \\ ${ }^{4}$ Institute of Nano Science and Technology, University of Science and Technology of China, Hefei, China \\ ${ }^{5}$ The Abdus Salam International Centre for Theoretical Physics (ICTP), Trieste, Italy \\ ${ }^{6}$ Department of Physics, Hazara University Mansehra, Pakistan \\ ${ }^{7}$ Department of Electronics Quaid-i-Azam University, 45320 Islamabad, Pakistan ${ }^{\dagger}$ \\ ${ }^{8}$ Department of Physical Science and Technology, Sichuan University, Chengdu, Sichuan, China
}

\begin{abstract}
The laser differential equations are used to transform them into identical coupled maps. Valuable results are deduced during analytical and numerical studies on cavity loss. Phase and spatiotemporal synchronized attractors are observed via quasi-chaos under a certain range of controlling parameters, and symmetry breaking of chaotic attractors due to collision with their basin boundaries, and transpire differently from the previous attractors. During the numerical simulation, it is found that the sequence of repeated strange attractors if the coupling strength further increases, which are orthogonal mirror images (the dynamics of the system is the same at different values of controlling parameters). Moreover, it can help us to predict future problems and their solutions based on current issues, if we develop this model in more general.
\end{abstract}

PACS numbers: 05.45.Gg, 05.45.Pq, 42.60.Jf

\section{INTRODUCTION}

In the early 1700, a famous scientist Christiaan Huygens discovered a phenomenon of synchronization between coupled pendulum clocks hanging from a common support. In many species of synchronous rhythms of cell apportionment in the organism inform the cell cycle oscillators and circadian are coupled [1]. The biological phaselocking observed in proliferating mouse fibroblasts accommodates synchronized oscillations between the circadian clock and cell cycle [2]. Synchronization of dynamical systems is crucial and received a substantial range of curiosity in recent research. Especially, mathematical modeling to address multispectral imaging of single live cells in proliferating mouse fibroblasts [2], secure communication which depends on an array of electronic circuits and semiconductor lasers [3], synchronized mirror image orthogonal attractors when the coupling strength of the coupled laser's system is complex values [4]. Synchronization in dynamical systems depends on the coupling strength, which does work as feedback and causes stable states. If the values of coupling delay adjust with cavity loss, it can provide ideal results as well as get new complex phenomena. The system goes from synchronized phase to irregular trajectories when the values of coupling strength increase beyond the critical domain and cause the crisis [5]. The crisis mean basin boundaries of the strange attractors collide and another state of the

\footnotetext{
*Electronic address: mabdul@mail.ustc.edu.cn

$\dagger$ Electronic address: farhan.saif@qau.edu.pk
}

system which may be stable or unstable depends on the value of controlling parameters.

To introduce the cavity decay rate in our dynamical model, we employed the thermal fluctuation theorem. After developing the mathematical model for cavity loss in the form of heat energy, noise, and thermal fluctuations which can cause instability in different lasing systems and experiments. We will be able to know which parameters at what value gives the desired results. If $\mathrm{V}$ is the volume of the cavity and $\mathrm{l}$ is the length and span $\mathrm{S}$ distinct modes. There must be damping inside the cavity, if the field is transmitted through the mirrors. To analysis before and after existing damping which causes to reduce the amplitude of the field propagating into the cavity. We drive the quality-factor of the ring-cavity by utilizing some fundamental equations which base on atom-field and mirror interactions.

In this paper, we develop a mathematical laser system model which consists of coupling delay as well as cavity loss. The main purpose of this coupled semiconductor laser model is to study and investigate the complex phenomena in photonic devices due to coupling delay and cavity loss. The emerging of complex demeanor, synchronization chaotic attractors in a spatially distributed and deterministic chaos seen during our numerical and analytical calculation of the coupled laser system at the weak values of the coupling strength. The dynamical model described here serves as a distinct concept for the entrainment behavior between the strange attractors of coupled nonlinear oscillators. The chaotic attractors remain orthogonal phase entrained synchronized at the fixed values of coupling delay $\gamma, \&$ cavity loss $\gamma_{c}$, and at different value of $\lambda$, which is the growth rate of our 


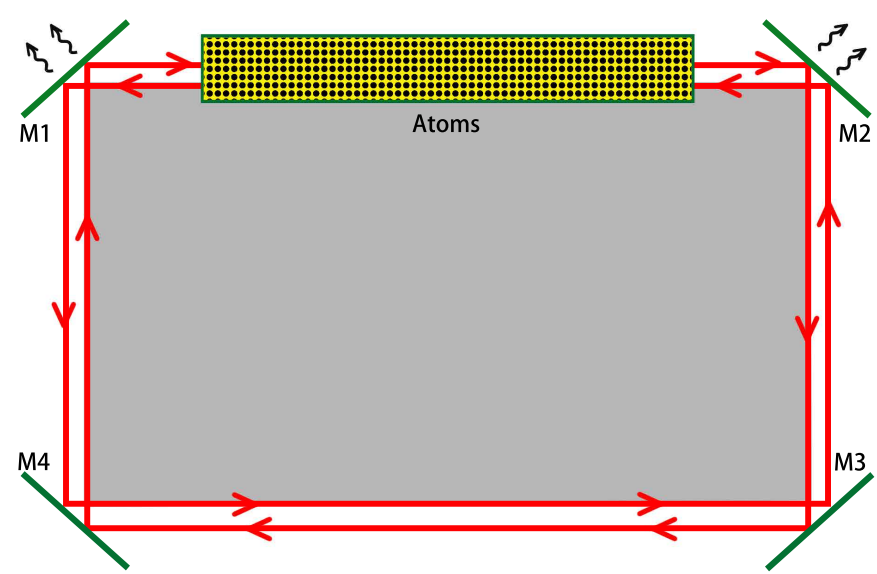

FIG. 1: The schematic diagram of ring cavity in which two waves are propagating in clockwise and anti-clockwise directions.

dynamical system. The trajectories of coupled units remain synchronized even the motion of the whole network is chaotic. Synchronization and symmetry break when the values of coupling delay are strong and the whole trajectory in phase space behaves like irregular as well as turbulence chaos [7, 8]. Moreover, in the absence of coupling decay, each chaotic attractor behaves like a single longitudinal and transverse mode $[9,10]$.

\section{TWO-MODE COUPLED LASERS}

A ring cavity contains excited atoms with decay rate $\gamma_{1}$, in which the two waves are traveling in clockwise and counter-clockwise directions [11, 12], as shown in Fig. 1. The reflectivity of mirrors $M_{1}$ and $M_{2}$ is $R$ and a part of the waves transmitted through these two mirrors. At the same time, the mirrors $M_{3}$ and $M_{4}$ have $100 \%$ reflectivity, which causes the reflected amplified light back to interact with atoms. A complete derivation of a singlemode laser logistic map is driven by utilizing the interacting Hamiltonian in [13]. Since the two electric waves are propagating in opposite directions and they interact with atoms which are kept inside the ring cavity, atom-atom interaction, their collision, loss of energy in the form of heat, and other forms. Therefore, a simplified mathematical formulation of two waves that are propagating inside the ring cavity is written as [14-16],

$$
\begin{aligned}
& \frac{d E_{1}}{d t}=\left[A-C_{1}-B\left(\left|E_{1}\right|^{2}+\xi\left|E_{2}\right|^{2}\right)\right] E_{1}, \\
& \frac{d E_{2}}{d t}=\left[A-C_{2}-B\left(\left|E_{2}\right|^{2}+\xi\left|E_{1}\right|^{2}\right)\right] E_{2},
\end{aligned}
$$

These equations are also called nonlinear coupled laser's dynamical equations, and represented in the form of differential electric field $E_{1}$ and $E_{2}$. Here, A, B and C are the Einstein gain coefficient, nonlinear saturation and loss of the system respectively and defined as,

$$
\begin{array}{r}
A=\omega \bar{\eta}\left(p_{2}-p_{1}\right) \mu_{12}^{2} T / 2 \varepsilon_{0} \hbar \\
B=2 \omega F\left(p_{2}-p_{1}\right) \mu_{12}^{4} T^{3} / 2 \varepsilon_{0} \hbar^{3}, \\
C=\sigma_{i} / 2 \varepsilon_{0},
\end{array}
$$

Here, $\xi$ is defined as,

$$
\xi=\frac{1}{\left[1+\left(\Delta \omega T_{1}\right)^{2}\right]},
$$

here, $\Delta \omega$, and $T_{1}$ are the detuning and natural lifetime of laser transitions, respectively [15]. Furthermore, $\xi$ for 2,1 , and $<1$ corresponds to an homogeneously broadened gain medium, an inhomogeneously broadened gain medium at the line center, and a detuned inhomogeneously broadened laser respectively.

To get the desired dynamical model, we need to multiply $2 E_{1}^{*}$ and $2 E_{2}^{*}$ by Eq. (1), and the simplification provides us the following results,

$$
\begin{aligned}
& \dot{I}_{1}=2\left(A-C_{1}\right)\left[1-\frac{B}{\left(A-C_{1}\right)}\left(I_{1}+\xi I_{2}\right)\right] I_{1}, \\
& \dot{I}_{2}=2\left(A-C_{2}\right)\left[1-\frac{B}{\left(A-C_{2}\right)}\left(I_{2}+\xi I_{1}\right)\right] I_{2},
\end{aligned}
$$

here, $I_{i}=\left|E_{i}\right|^{2}$ and $i=1,2$, we take only the real part $\operatorname{Re}\left(E_{i}\right)$ of the complex equations. Since the intensity is directly proportional to the number of photons, therefore, the above equations can be written as,

$$
\begin{aligned}
& \dot{x}=\dot{n_{1}}=2\left(A-C_{1}\right)\left[1-\left(n_{1}+\xi n_{2}\right)\right] n_{1}, \\
& \dot{y}=\dot{n_{2}}=2\left(A-C_{2}\right)\left[1-\left(n_{2}+\xi n_{1}\right)\right] n_{2} .
\end{aligned}
$$

In the absence of coupling strength, the fractions $\left(\frac{B}{\left(A-C_{i}\right)}\right)^{-1}$ are represented as steady-state photon numbers and have too small value nearly equal to zero, so we can cut-down this factor from the Eqs. (6) and get the simplified model. For further simplification, we are defining a differential term as $\frac{d x}{d t}=\frac{x_{j+1}-x_{j}}{\Delta t}$, and the controlling parameters are $\left(A-C_{i}\right) \Delta t=\lambda_{i}$. Here $\Delta t$ is the cavity round trip time which represents the period of the waves propagating in the direction of clockwise and anticlockwise $t^{+}$and $t^{-}$respectively. Finally, we derived the general form of laser logistic coupled maps,

$$
\begin{aligned}
x_{n+1} & =x_{n}+2 \lambda_{1} x_{n}\left[1-\left(x_{n}+\xi y_{n}\right)\right], \\
y_{n+1} & =y_{n}+2 \lambda_{2} y_{n}\left[1-\left(y_{n}+\xi x_{n}\right)\right] .
\end{aligned}
$$

The coupled logistic equations for a two-mode laser system with cavity decay are described via thermal fluctuations in the next section.

\section{ELECTROMAGNETIC FLUCTUATION IN A RING CAVITY}

Thermal fluctuation effects in a cavity are fundamental phenomena, which help to improve the characteristics 
of the system. Since, the cavity consists of the spacer, isotropic and homogeneous medium, mirror-substrate, and reflective coating, as shown in Fig. (1). The frequency dependent dielectric $\epsilon(\omega)$ and magnetic permeability $\mu(\omega)$, are defined as,

$$
\begin{gathered}
\vec{D}(\omega)=\epsilon(\omega) \vec{E}(\omega) \quad ; \quad \vec{B}(\omega)=u(\omega) \vec{H}(\omega) \\
\vec{E}(\omega)=\int e^{i \omega t} \vec{E}(\vec{r}, t) d t, \text { and } \\
\vec{E}(\vec{r}, t)=E(t) e^{-i \omega t} \mu(\vec{r})+c . c .
\end{gathered}
$$

Since $\mathrm{V}$ is volume of the cavity, $N_{2}$ is the number of excited atoms and A Einstein gain coefficient. Thus, the total rate of spontaneous emission of photons is $A N_{2}$, if these emissions span $S$ distinct cavity modes, then all exist within the natural linewidth $\mathrm{A}$, and the density of cavity modes is $V / 4 \pi^{3}$, therefore,

$$
S \approx \frac{V}{4 \pi^{3}}\left(\frac{\omega_{0} A}{c^{3}}\right) 4 \pi=\frac{\omega_{0} V A}{\pi^{2} c^{3}}
$$

Here, $\omega_{0}$ and $\mu(\vec{r})$ are the frequency of atomic transition, and the eigenfunction, respectively. The lost of photons from the given cavity mode is,

$$
\frac{n \pi^{2} c^{3}\left(N_{2}-N_{1}\right)}{\omega_{0}^{2} V} \geqslant \frac{n}{T_{L}}
$$

The strike rate of the cavity photons with the mirror is defined as, $1 / T_{L}=(c / 2 l)(1-R)$, with the help of eq. (10), for one cavity mode, the laser oscillation condition is driven as,

$$
N_{2}-N_{1} \geqslant \frac{\omega_{0}^{2} A}{2 \pi^{2} c^{2}}(1-R)=\frac{2 A}{\lambda^{2}}(1-R),
$$

where, $\lambda$ is the wavelength, and the atomic populations inversion is directly proportional to the transmissivity of the output mirror of the cavity, which required for laser action occur. To describe the characteristic of cavity, we can drive the formula from the above equation, which is called cavity Q-factor,

$$
Q=\frac{2 \pi l / \lambda}{(1-R)}=\frac{\sigma}{2 \epsilon_{0}}
$$

The quality factor of the cavity helps us to measure the entire damped phase which elapses before the oscillation reduces in amplitude. The Rabi frequency which is represented as field amplitude, which can be positive or negative,

$$
\Omega=2\left|\mu_{12}\right| \quad|E(t)||\mu(\vec{r})| / \hbar
$$

For the special case of laser instability, in which the amplitude $E(t)$, and transition dipole moment $\mu_{12}$ are containing the real values, the equation of motion for the field is,

$$
\begin{aligned}
\frac{d \Omega}{d t}+\frac{\sigma}{2 \epsilon_{0}} \Omega=\frac{\mu_{12}^{2} \omega \eta}{2 \epsilon_{0} \hbar}\left[\frac{\sin (k L / 2)}{(k L / 2)}\right]{ }^{\prime} & \text { or } \\
\frac{d \Omega}{d t}+\gamma_{c} \Omega & =\omega g \gamma_{2}^{\prime},
\end{aligned}
$$

here, $\gamma_{c}=\sigma / 2 \epsilon_{0}$, and $g=\frac{\mu_{12}^{2} \omega \eta}{2 \epsilon_{0} \hbar}\left[\frac{\sin (k L / 2)}{(k L / 2)}\right]$ are the cavity damping rate in the absence of threshold level, and the coupling rate between field and atoms, respectively. The coupling-parameter itself causes instability, symmetry breaking, and time-delay which directly approach dissipation and effect on amplitude and propagation as well as vanish the synchronization among the strange attractors when the cavity loss has random values. For precisely investigation of laser instability and chaos, we need to solve the above equation with the Bloch equations in the rotating frame. Our concern is to determine the damping parameter, so introducing the phenomenological damping terms $\gamma_{\perp} \gamma_{2}^{\prime}$ and $\gamma_{\|} \gamma_{3}^{\prime}$, and atomic inversion $\gamma_{3}^{\prime}$. We will get three coupled laser equations, in which $\gamma_{e}^{\prime}$ represents the equilibrium value of $\gamma_{3}$ in the absence of the field. The damping parameter is calculated at the laser threshold, in which the gain rate is equal to the lose rate, i.e., $\gamma_{c}=\frac{\omega g \hat{\gamma}_{e}}{\gamma_{\perp}}$. For the further simplification of the problem from three variables to one we eliminate the possible certain instabilities. For the complete derivation and physical significants, please see the chapters 15 and 18 of reference 12 . Therefore, modify version of Eq. (6) with cavity lost can be defined as,

$$
\begin{aligned}
& x_{n+1}=x_{n} e^{-\gamma_{c}}+2 \lambda_{1} x_{n}\left(1-x_{n}\right)-\gamma x_{n} y_{n}=f_{\lambda_{1}, \gamma}^{(1)}\left(x_{n}, y_{n}\right), \\
& y_{n+1}=y_{n} e^{-\gamma_{c}}+2 \lambda_{2} y_{n}\left(1-y_{n}\right)-\gamma x_{n} y_{n}=f_{\lambda_{2}, \gamma}^{(2)}\left(x_{n}, y_{n}\right),
\end{aligned}
$$

where, $\lambda_{i}$, and $\gamma=2 \xi \lambda_{i},(i=1,2)$ are the characteristic three paramount parameters. The parameter $\gamma_{c}$ is the cavity decay rate for the two modes laser's system. If we take the ideal case of the cavity decay rate and set $\gamma_{c}=0$, we obtain the dynamical system outward of the cavity decay rate. A mapping of bilinear and linear coupling terms has been shown to exhibit complicated dynamical behavior including quasi-periodicity, phase locking, intermittency, period adding, long-lived chaotic transitions, and then periodicity [17-19]. Here, we explain the necessary terms and their symbols used in our later discussion. There occurs a fascinating behavior of the coupled logistic maps (15) for various values of $\lambda_{i}$ and $\gamma_{i}$. The key to understanding the structure of these equations in $X Y$-space is a careful analysis of the fixed points of the mapping functions as well as their iterations. Since the functions $f^{(1)}$ and $f^{(2)}$ are symmetrical, we expect symmetrical behavior in $x$ and $y$.

By fixing $\gamma, \gamma_{c}$, and varying $\lambda$ we have found evidence for a boundary crisis in our system, like or similar to the Henon's map by Grebogi, Ott, and Yorke [18, 20]. A boundary crisis occurs in our system through the collision of the chaotic attractor with a basin boundary that 

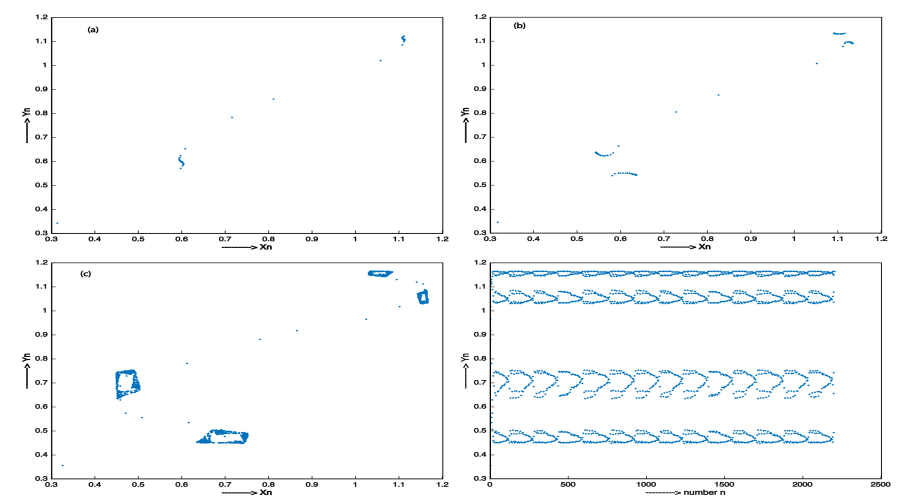

FIG. 2: Phase space $\left(x_{n}, y_{n}\right)$ is plotted by solving Eq. (15), for $\gamma_{2}=0.09$, and (a) $\lambda_{i}=1.2411$, which shows that $2 \mathrm{P}$, and (b) $\lambda=1.2599$ periodic behaviour of $4 \mathrm{P}$, and (c) $\lambda=1.31290$, which is behaving like a quasi-periodicity. We note that the system displays a $4 \mathrm{P}$ to $8 \mathrm{P}$ behavior through period bifurcation using crises. In the plot on the right side, we present $y$-axis of the phase space as a function of the number of iterations $n$.

separate it from the several other coexistent periodic attractors, besides with another chaotic attractor [21, 22]. An increase of $\lambda_{i}$ beyond its critical value for the onset of crises results in the disappearance of the chaotic attractors and its basin, while the basins of the remaining attractors undergo a sudden expansion.

We report the chaotic behavior in our system corresponding to various values of the parameters. The behavior of our dynamical system is classified into two cases: In the first case, we fix $\gamma_{2}=\gamma=\gamma_{c}=0.09$, at the same values, and vary $\lambda_{i}$ over the range $0.001 \leq \lambda_{i} \leq 0.25$ for $x_{0}=0.1$ and $y_{0}=0.11[27,33]$. At $\lambda_{i}=0.25$, the trajectory in phase space $(x, y)$ converges to a fixed point. The asymptotic character of the solution is typically $1 \mathrm{P}$. For the $\gamma_{2}$ fixed value, the system shows oscillatory behavior between QP and $2 \mathrm{P}$ characters up to $\lambda_{i}=1.25$. There is still $2 \mathrm{P}$ character for $\gamma_{2}$, and in the interval $\lambda_{i} \in[1.0444,1.2511]$. At the upper range of this interval, it shows $2 \mathrm{P}, 4 \mathrm{P}$, and $\mathrm{QP} 4$ Torus, respectively, at $\lambda_{i}=1.2411,1.2599$, and 1.31290. Periodic bifurcation phenomena are also observed, as shown in Fig. 2. There is no chaotic behavior seen, but our coupled system oscillates between $4 \mathrm{P}$ to $8 \mathrm{P}$ through quasi-periodicity. It is a clarion that if the coupling strength and decay rate remain fixed and $\lambda_{i}$ is increased, the periodicity fuses and comes out of the crisis.

- For $\lambda_{i}=1.31370$ and $\gamma_{2}=0.09$, the four chaotic attractors grow as any parameter of the coupled logistic equation increases. They are synchronized (mirror images of each other) as well as orthogonal, and the trajectory in phase space converges to $8 \mathrm{P}$ via $\mathrm{QP}$. When $\lambda_{i}=1.31470$, a transition from $8 \mathrm{P}$ to $16 \mathrm{P}$ occurs, which shows quasi periodicity in which each attractor displays a curious pattern before settling to the asymptotic $16 \mathrm{P}$ state as shown in Fig. 3(a). They also
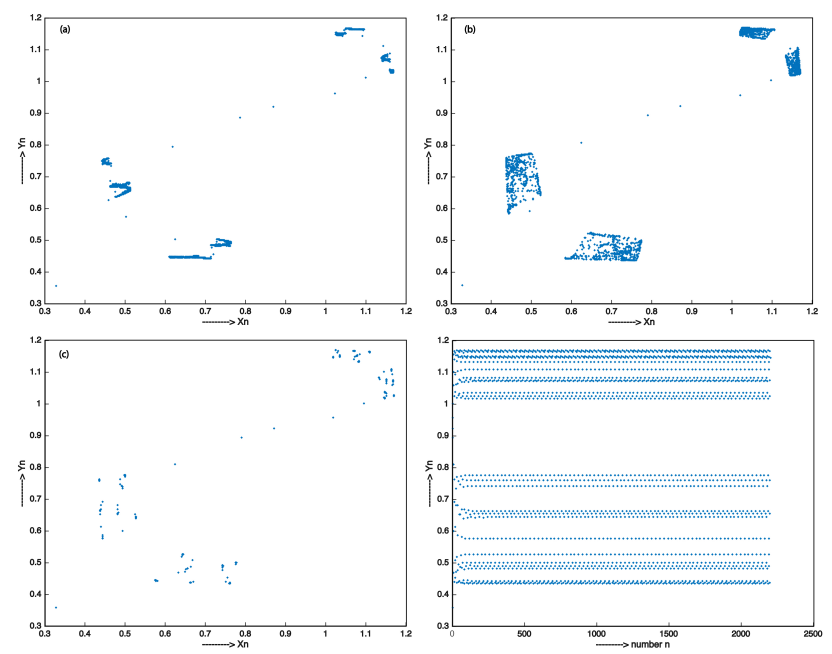

FIG. 3: Phase space $\left(x_{n}, y_{n}\right)$ is plotted by solving Eq. (15) for $\gamma_{2}=0.09$, and (a) $\lambda_{i}=1.31670$, which has period 16 ; (b) $\lambda_{i}=1.31990$, which show quasi-stable behaviour; (c) $\lambda_{i}=1.3209$, stable of $24 \mathrm{P}$. The plots on the right side of (c) show stability and (b) shows the intermittency state of the system. Isolated points are part of transient evolution. Here trajectory and periodicity of our system dramatically change because of crises. The exterior crises are observed between two trajectories.

fluctuate and expand due to crises during the interval $\lambda_{i} \in[1.31470,1.3166]$ and we observed the state of the system change, which generates a mirror image of laser attractors beyond this interval. At $\lambda_{i}=1.31670$, the transient solution again shows a curious pattern of torus before going to the periodic regime. At $\lambda_{i}=1.31770$, the lasing system shows Quasi-chaotic behavior through QP and the laser attractors in phase space have the exact mirror image of each other. In laser chaos, there exist inner crises, a boundary crisis where a strange attractor collides with unstable fixed points on the boundary of the basin of attraction, causing the disappearance of both [20, 23], as shown in Fig. 3(b).

During the numerical simulation, it is observed, when a boundary crisis happened, the unstable fixed points disappear, and the shape of the strange attractor change causes unstable (chaotic behavior) as the parameters increases. In the presence of cavity delay, the behaviour of our newly developed model reverses as we studied the model without cavity delay i. e, when $\lambda_{i}=1.3209$ each period (line) diffuses from eight to four, and finally into $24 \mathrm{P}$ (24P and 24 attractors) and the chaotic attractors remain mirror images to each other, as shown in Fig. 3(c). The strange attractors remain phaseentrained, orthogonal synchronized (a kind of entangled attractors which depend on coupling strength and cavity delay rate) and stable to quasi-stable state for the intervals, where $\lambda_{i} \in[1.2522,1.3]$ and $[1.3,1.3209][24-$ $26]$, we can say about this situation of the system showing spatio-temporal fractals. Furthermore, chaotic attractors 

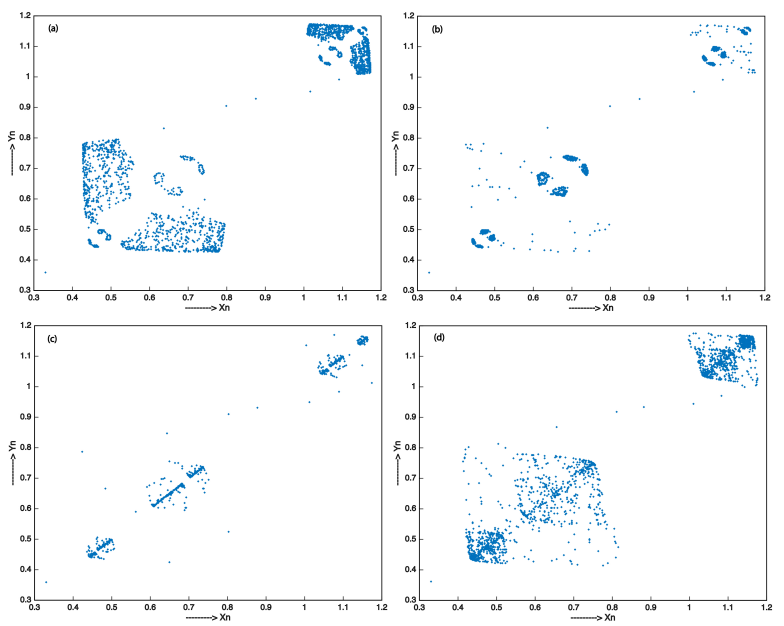

FIG. 4: Phase space $\left(x_{n}, y_{n}\right)$ is plotted by solving Eq. (15) for $\gamma_{2}=0.09$, and (a) $\lambda_{i}=1.3263$, which shows quasichaotic behaviour (b) $\lambda_{i}=1.3265$ represent stable system, (c) $\lambda_{i}=1.3295$, having $24 \mathrm{P}$, and (d) $\lambda_{i}=1.3343$, which shows chaotic behaviour. As we mentioned earlier, isolated points in (a) to (d) are part of transient evolution. Therefore, our dynamic system is going from a stable state to an unstable state through QP because of hysteresis crises.

are mirror images of each other in the interval [1.3177, 1.3222], while the trajectory changes at $\lambda_{i}=1.384$ if the increases above this value, we get different forms of trajectory due to boundary crises, which is called symmetry breaking i. e, chaotic attractors are not mirrored images $[24,25]$. The band emerging in a coupled logistic map is the coalesce $(24 C \rightarrow 8 C \rightarrow 4 C)$ chaos to chaos analogous [27-29]. Therefore, the periodicity of the coupled lasing system oscillates (4P to $24 \mathrm{P}$, each line of period 4 bifurcate, then multi-bifurcation behavior at $\left.\lambda_{i}=1.3209\right) 4 \mathrm{P}$ to $8 \mathrm{P}$ to $24 \mathrm{P}$ through $\mathrm{QP}$ and chaotic, as shown in Fig. 3, Which gives the information about the dynamical at different parameters.

As we are increasing the value of $\lambda_{i}$, and corresponding to it, we are observing the dynamics of our system in phase space. The coupled logistic maps become consequential beyond $\lambda_{i}=1.31$ and the trajectory dynamically changes. For $\lambda_{i}=1.3263$ and $\gamma_{2}=0.09$, the dynamics of our system in phase space behaves like fractal mirror images and quasi-stable system. The isolated points in phase space are part of the transition, i.e, as the parameters increase, the basin boundaries of the stranger attractors expand and cause hysteresis crises, as shown in Fig. 4(a-d). For $\lambda_{i}=1.3265$, our dynamical system goes to stable and gets the mirror image fractals. The isolated and occupied points in the phase space play a paramount role in the dynamical systems [30-32]. However, for $\lambda_{i}=1.3199$ to 1.3290 , the system remains in a quasi-state and then converges in exactly 24 iterations at $\lambda_{i}=1.3295$. Chaoticity and periodicity (24P) oscillate in the interval $[1.3209,1.3295]$ and some other complex phenomena have also happened $[23,33]$. The cou- pled logistic maps show the chaotic behavior via a quasistable-state at $\lambda_{i}=1.3343$. At the upper range of the above-described interval, our system shows chaotic behavior through a nodal period, QP and 24P. Lasing system shows chaotic behavior for a small interval of time and comes back to its initial state when the coupling strength reaches its maximum value for small values of parameters.

The torus represents the quasi-periodic behavior which consists of two variables amplitudes and phase even if we take the Poincare section. The described dynamical system moves to the chaotic region via quasistable state with increasing value of the growth rate of coupled maps [17]. We analysis the Poincare section of two dynamical discrete systems, the sequence from stable state to chaos is characterized as: A stable limit cycle corresponds $2 \mathrm{P}$. If the value of $\lambda$ increase beyond the critical limit, the limit-cycle goes to unstable and bifurcates into four-loop limit-cycles and emerges into an eight-loop torus through a Hopf bifurcation. In the coupled dynamical system which possess the coupling strength negatively corresponds synchronization and four orbits in phase and their state can be explained as the four intermittencies periodic behaviors show when the four characteristic frequencies of the torus are in the ratio of two small integers [33-35]. When the values of $\lambda_{1}$ and $\lambda_{2}$ are different from each other, the four synchronized attractors convert into two orbits, which show orthogonal mirror images.

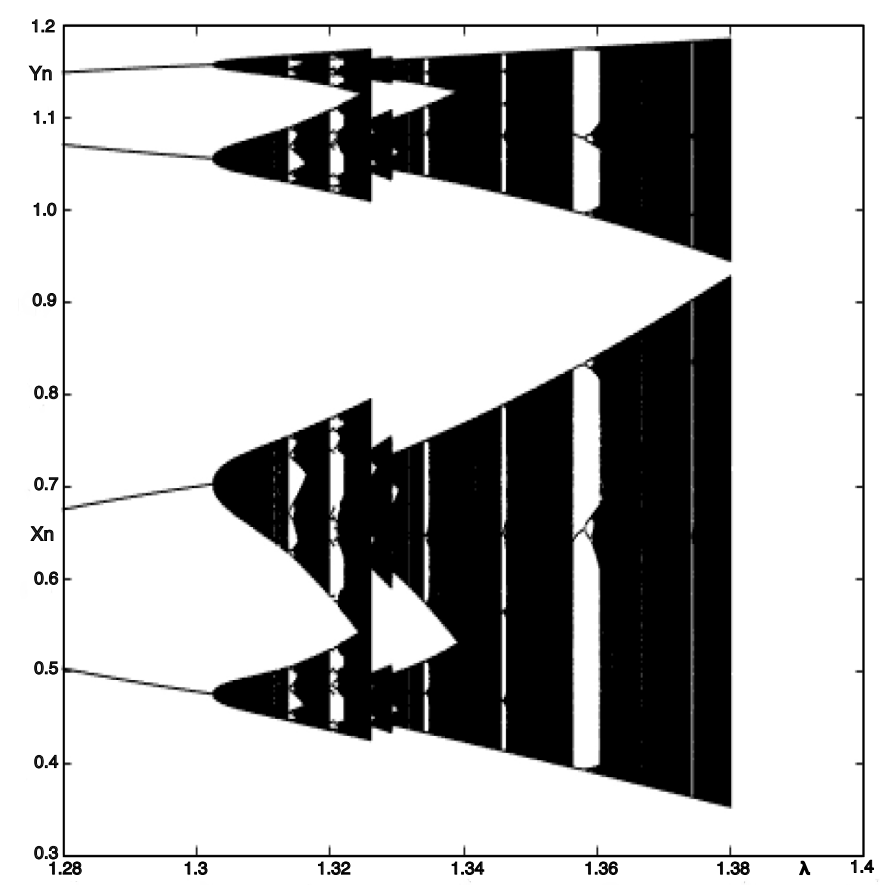

FIG. 5: Bifurcation diagram for the coupled lasers Eq. (15) with $\lambda_{1}=\lambda_{2}$ range from 1.28 to 1.38 and $\gamma_{2}=0.09$. For each value of $\lambda_{i}$ we used the final point of the previous $\lambda_{i}$ value and 4000 iterates are plotted. This shows the period-doubling sequence to chaotic regions. 
The beauty of our dynamical system is exhibited including multibasin strange attractors, period-doubling bifurcation, Hopf-bifurcation, and so on. The perioddoubling bifurcation sequence of coupled maps is a sticking mechanism, which sketches the whole distinct dynamics of any dynamical model. The coupled equations have only two equations which represent a very simple system but reveal complicated dynamics in the phase space, i. e., an avalanche of period-doubling routes to chaos were seen in our lasing system as presented by [36-38]. A twomode ring cavity laser's system is also an interferometer whose output is investigated through the controlling parameters, i. e., cavity loss, coupling delay, collision of atoms to atoms, and field interaction. We analysis our model via the wide range of two parameters, i. e., $\lambda_{i}$, and $\gamma_{2}$, where $\lambda$ is chosen to vary in a small neighbouring of $x_{n}, y_{n}$ in the plotting of the bifurcation diagram and observed numerically period-three cycles to chaos as shown in Fig. 5, the same as [39-42].

\section{CONCLUSION}

This paper provides a singularity study of coupled laser equations which consists of coupling delay as well as cavity loss. The numerical results construe the dynamics of the lasing system transition from stability to chaos through the torus. It is observed that a nonlinear feedback loop helps to control and stabilize the laser system or beam intensity. Many complex dynamics and behaviors are noticed when two signals are propagating with the finite speed in the opposite direction and their interactions with different things as well as the time de- lay. To study the collective behavior of a coupled system that can play the role of a testbed system and helps to fabricate well-defined working devices. If the dynamical system is evolved under the influence of quasi-state and only the growth rate is increasing, causing the unstablestate with different phase-space dynamics. The system moves to another newly chaotic regime which is different from the previous if and only if the value of growth rate increases. In our model, we also observed during our numerical simulations, the symmetry breaking, synchronization vanishing mirror image $\&$ phase-entrained disappeared when the values of one controlling parameter increase beyond the critical range, among the different parameters.

In case of varying the values of cavity decay rate $\gamma_{1}$ and coupling delay $\gamma_{2}$, the testbed-system remains stable and synchronized for the large value of growth rate. The cyclic crisis phenomena are paramount in the coupled dynamical system.

\section{ACKNOWLEDGEMENT}

This project is supported by CAS-TWAS and Sichuan University (The Fundamental Research Fund for the Central Universities), I am also grateful for the staff who helped and supported me when I was writing this article.

\section{AUTHOR CONTRIBUTION STATEMENT}

All authors contributed equally to the paper
[1] Arkady Pikovsky, Michael Rosenblum, and Jurgen Kurths, Synchronization, A universal concept in nonlinear sciences (Cambridge University Press, Cambridge, 2001).

[2] Celine Feillet, Peter Krusche, Filippo Tamanini, Roel C. Janssns, Mike J. Downey, Patrick Martin, Michele Teboul, Shoko Saito, Francis A. Levi, Till Bretscheirder, Gijsbertus T. J. van der Horst, Franck Delaunay, and David A. Rand, PANS 11, 98289833 (2014).

[3] W. Kinzel, A. Englert, M. Zigzag, and I. Kanter, Phy. Rev. E. 97, 056207 (2009).

[4] M. Abdul, and F. Saif, 6th chaotic Modeling and Simulation International conference 11 - 14 June 2013 Istanbul, Turkey: Synchronized Attractors and Phase Entrained Chaos (2013).

[5] Miguel C. Sorian, Jordi Garcia-Ojalvo, Claudio R Mirasso, and Ingo Fisher, Complex photons: Dynamics and applications of delay-coupled semiconductors lasers, Rev of Mod Phy. 85, 421 (2013).

[6] News, Health issues, Discussion of COVID-19 with experts and data analysis from, 2019 to (2021).

[7] Shuguang Guan, G. W. Wei, and C. H Lai, Phys. Rev.
E. 69, 066214 (2004).

[8] Sudeshna Sinha, Phy. Rev. E 66, 016209 (2008).

[9] Herbert G. Winful and Lutfur Rahman, Phys. Rev. Lett. 65, 13 (1990).

[10] A. Englert, S. Heiligenthal, W. Kinzel and I. Kanter, Phys. Rev. E. 83, 046222 (2011).

[11] W. T. Silfast, Laser Fundamentals Second Edition (Cambridge University Press, Cambridge, 2004).

[12] M. Mandel and E. Wolf, Optical Coherence and Quantum Optics (Cambridge University Press, Cambridge, 1995).

[13] M Abdul, Umer Farooq,Jehan Akbar, and F. Saif, Discrete Dynamical Laser Equation for Critical Onset of Bistability, Entanglement and Disappearance, Laser Physics 28, 6 (2018).

[14] W. E. Lamb, Phy. Rev. 134, 1429. (1964).

[15] M. M. Tehrani and L. Mandel, Mode competition in a ring laser at line center, Opt. Commun. 16, 16 (1976).

[16] R. Blumel, and W. P. Reinhardt, Chaos in Atomic Physics (Cambridge University Press, Cambridge, 1997).

[17] Kunihko. Kaneko , Progress of Theoretical Physics 69, 1427 (1983).

[18] G. Tanaka, M. A. F. Sanjuán, and K. Aihara, Phys. Rev. E. 71, 016219 (2005). 
[19] Maryam Ghorbani, Mayank Mehta, Robijin Bruinsma, and Alex J Levine, Phy. Rev. E 85, 021908 (2012).

[20] C. Grebogi, E. Ott and J. A. Yorke, Phys. Rev. Lett. 48, 1507 (1982).

[21] Xingyuan. Wang and Chao Luo, Inter. Jour. of Mode. Phys. B. 22, 427 (2008).

[22] V. Astakhov, A. Shabunin, T. Kapitaniak and V. Anishchenko, Phys. Rev. L. 79, 6 (1997).

[23] V. Astakhov, and A Shabunin, Phys. Rev. E. 63, 056212 (2001).

[24] Falconer K. J, The Geometry of Fractal Sets. (Cambridge University Press, Cambridge, 1985).

[25] Arne Jakobsen, Physica. D 237, 3382 (2008).

[26] M. Rosenblum A. Pikovsky, and J. Kurths, Phy. Rev. Lett. 76, 1804 (1996).

[27] Yan. Gu, Mingwhei. Tung, Jian-Min. Yuan, Da Hsuan. Feng and Lorenzo. M. Narducci, Phys. Rev. Lett. 52, 9 (1984).

[28] Igor Franovic, Kristina Todorovic, Nebojsa Vasovic, and Nikola Buric, Phy. Rev. Lett. 108, 094101 (2012).

[29] M. Rosenblum A. Pikovsky, and J. Kurths, Europhy. Lett. 34, 165 (1996).

[30] H. Fujisaka, and T Yamada, Prog. Theor. Phy. 69, 32 (1983).

[31] L. M Pecora, and T. L Carroll, Phy. Rev. Lett. 64, 821 (1990); M. de Sousa Vieira, A. J. Lichtenberg, and M. A.
Lieberman, Int. J. Bifurcation Chaos Appl. Sci. Eng. 1, 691. (1991).

[32] Garfinkel A, Self-organizing systems(Plenum Press, New York, 1987).

[33] J. M. Yuan, M. Whei. Tung, D. H. Feng, and L. M. Narducci, Phys. Rev. A. 28, 1662 (1983).

[34] Zhanybai T. Zhusubaliyev, and Erik Mosekilde, Phys. Lett. A. 373, 946 (2011).

[35] Parker. T. S and L. O. Chua, Practical Numerical Algorithms for Chaotic System. (New York: Springer-Verlag, New York, 1989).

[36] K. Ikeda, and K. Matsumoto, Physica Amstredam 29D, 223 (1987).

[37] K. Ikeda, K. Otsuka and K. Matsumoto, Prog. Theor. Phys. Suppl. 30, 295 (1989).

[38] Briggs. J, and Peat. F. D, The turbulent mirror (Harper and Row Press, New York, 1989).

[39] J. P Goedgebuer, Laurent Larger, and Herri Porte, Phy. Rev. Lett. 80, 2249 (1998).

[40] J. P Goedgebuer, Laurent Larger, Herri Porte, and F. Delorme, Phy. Rev. E. 57, 2795 (1998).

[41] Rossler. O. E. The chaotic hierarchy. In A Chaotic Hierarchy. (, Singapore, 1991).

[42] Wiggins. S, Global Bifurcations and Chaos: Analytical Methods. (New York: Springer-Verlag, New York, 1988). 